\title{
Impact of Chemotherapy-Induced Nausea and Vomiting on Quality of Life in Indonesian Patients With Gynecologic Cancer
}

\author{
Dyah Aryani Perwitasari, MSi, * + Jarir Atthobari, PhD, $\dagger$ Mustofa Mustofa, Dr, $\uparrow$ \\ Iwan Dwiprahasto, PhD, † Mohammad Hakimi, PhD, § Hans Gelderblom, MD, PhD,// Hein Putter, PhD, I \\ Johan W.R. Nortier, MD, PhD,// Henk-Jan Guchelaar, PhD, $\neq$ and Ad A. Kaptein, PhD\#
}

\begin{abstract}
Background: Quality of life (QoL) has become a major outcome in the treatment of patients with cancer. This study is aimed at examining the impact of chemotherapy-induced nausea and vomiting on QoL of patients with gynecologic cancer in Indonesia.

Methods: Chemotherapy-naive patients with gynecologic cancer, who were treated with cisplatin at a dosage $50 \mathrm{mg} / \mathrm{m}^{2}$ or higher as monotherapy or as part of combination chemotherapy regimens, were recruited in the Oncology Department, Dr. Sardjito Hospital, Yogyakarta, Indonesia. Quality of life was assessed by using the Indonesian version of the European Organization for Research and Treatment for Cancer of Quality of Life Questionnaire and Short Form-36, administered immediately before and on day 5 after chemotherapy administration. Patients used a daily diary to record nausea and vomiting during 5 days after chemotherapy.

Results: Most (74.9\%) of the 179 patients experienced delayed emesis during the 5 days after chemotherapy despite prophylactic use of antiemetics. The delayed nausea and emesis caused significant negative impact on patients' QoL. Nausea in the delayed phase caused negative effects on patients' QoL.

Conclusions: Patients reported a negative impact on the QoL of delayed emesis after chemotherapy. Poor prophylaxis of patients' nausea and vomiting after chemotherapy interferes with patients' QoL. Medical and behavioral interventions may help to alleviate the negative consequences of chemotherapeutic treatment in patients with gynecologic cancers treated with suboptimal antiemetics.
\end{abstract}

Key Words: Gynecologic cancer, EORTC QLQ-C30, SF-36, Indonesia, Nausea and vomiting, Chemotherapy

Received March 31, 2011, and in revised form August 23, 2011.

Accepted for publication August 29, 2011.

(Int J Gynecol Cancer 2011;00: 00-00)

In recent decades, quality of life (QoL) has increasingly become an important outcome in the treatment of patients with cancer. In QoL research, the impact of the illness itself and the cancer treatments are assessed from the patients' per- spective. One of the factors most seriously impacting patients' QoL during cancer treatment is chemotherapy-induced nausea and vomiting (CINV), especially when it is inadequately treated by antiemetic drugs. ${ }^{1}$ Patients may experience
*Pharmacy Faculty, Ahmad Dahlan University and $†$ Pharmacology and Therapy Department, Medicine Faculty, Gadjah Mada University, Yogyakarta, Indonesia; †Department of Clinical Pharmacy and Toxicology, Leiden University Medical Center, Leiden, The Netherlands; §Obstetric and Gynecologic Department, Medicine Faculty, Gadjah Mada University, Yogyakarta, Indonesia; and DepartCopyright (C) 2011 by IGCS and ESGO

ISSN: $1048-891 \mathrm{X}$

DOI: $10.1097 /$ IGC.0b013e318234f9ee ments of $\|$ Clinical Oncology, $\mid$ Medical Statistics, and \#Medical Psychology, Leiden University Medical Center, Leiden, The Netherlands. Address correspondence and reprint requests to Dyah A. Perwitasari, Pharmacy Faculty, Ahmad Dahlan University, Jl. Prof.

Dr. Soepomo, Janturan, Yogyakarta, Indonesia.

E-mail: D.A.Perwitasari@lumc.nl.

This study was supported by The Netherlands Organization for International Cooperation in Higher Education.

The authors declare that there are no conflicts of interest. 
acute or delayed CINV during cancer treatment. Acute CINV is defined as nausea and vomiting episodes, which persist during the first 24 hours after chemotherapy administration. ${ }^{2}$ Delayed CINV starts after the first 24 hours after chemotherapeutic treatment and may persist up to 120 hours. ${ }^{3}$ Patients who experience both acute and delayed CINV have worse QoL compared with patients with delayed CINV only or without acute and delayed CINV. ${ }^{1}$ By using an appropriate prophylactic antiemetic regimen, the incidence of CINV can be substantially reduced. However, despite the availability of highly effective antiemetic drugs, approximately $20 \%$ to $30 \%$ of the patients treated with highly emetogenic regimens still experience nausea and vomiting. This is caused by the presence of some risk factors, such as sex, age, and individual susceptibility. ${ }^{4}$ Health professionals often underestimate the incidence and prevalence of CINV. ${ }^{5}$

Most gynecologic cancer types are treated with highly emetogenic chemotherapeutics, such as cisplatin, carboplatin, paclitaxel, and cyclophosphamide when paclitaxel is not available. ${ }^{6}$ These drugs are reported to cause emesis in more than $90 \%$ of patients without prophylactic use of antiemetics $^{7}$ and may seriously impact QoL. In addition to CINV, the psychological distress after cancer diagnosis, especially issues concerning femininity, body image, sexuality, and reproduction also have been demonstrated to result in a decrease of the patients' QoL. ${ }^{5,8}$

The incidence of cervical cancer in Indonesia is 13.7 per 100,000 women in Indonesia. ${ }^{9}$ Despite this high incidence, information about the patients' QoL is still very limited. Therefore, we undertook this study to assess the QoL in gynecologic cancer patients after treatment with highly emetogenic chemotherapeutics, using the European Organization for Research and Treatment for Cancer of Quality of Life Questionnaire (EORTC QLQ-C30) as a disease-specific instrument and the Short Form-36 questionnaire (SF-36) as a generic instrument for QOL or functional status. Patients' QoL baseline condition was also determined to know the change of patients' QoL after treatment with chemotherapeutics. In addition, we addressed the impact of delayed CINV on these patients' QoL.

\section{METHODS}

\section{Patients}

The study sample consisted of chemotherapy-naive patients with cervical cancer $(n=120)$, ovarian cancer $(n=51)$, uterine cancer $(n=8)$, and vulva cancer $(n=7)$ in the Oncology Department of Dr. Sardjito Hospital, Yogyakarta, Indonesia. They were treated with cisplatin at a dosage of $50 \mathrm{mg} / \mathrm{m}^{2}$ or higher as monotherapy or in combination chemotherapy regimens. Patients with all diagnoses of gynecologic cancer and limited to pelvic or advanced stage of cancer were allowed to be included. Patients were referred by the general practitioners to the Dr. Sardjito Hospital, Yogyakarta, after the diagnosis was made. The staging procedures consisted of physical examination by a gynecologist and limited imaging when appropriate. Patients in the surgery consideration were treated according to the medical standard in Indonesia.
According to the standard of prophylactic antiemetic drug in the hospital, all patients were treated in the outpatient clinic by $8 \mathrm{mg}$ of intravenous ondansetron and $8 \mathrm{mg}$ of intravenous dexamethasone 1 hour before cisplatin administration as a prophylactic antiemetic regimen. All patients were also given oral metoclopramide $10 \mathrm{mg}, 3$ times a day, from day 2 to day 5, to prevent delayed emesis.

Patients aged 18 years or older with a Karnofsky index of $50 \%$ or higher were included. Patients with nausea or vomiting 24 hours before chemotherapy, use of other antiemetics than ondansetron and dexamethasone, use of benzodiazepines or neuroleptics, treatment with radiotherapy within 24 hours before the start of chemotherapy, and the use of opioids within the previous 2 weeks were excluded.

Patients' sociodemographic data were determined from their medical records. All patients gave informed consent. Data collection on demographics and baseline conditions was completed before administration of the chemotherapeutic drugs. Patients filled in the EORTC QLQ-C30 and SF-36 questionnaires 1 to 4 hours before chemotherapy and on day 5 after chemotherapy administration at the hospital. Not all of the patients could fill in the questionnaires by themselves and needed researcher assistance to explain some questions, that is, the questions about general health and general QoL in EORTC QLQ-C30. Patients were also asked to fill in a daily diary record to score the degree of nausea and the vomiting frequency during the 4 days after chemotherapy. The study was approved by the local ethics committee of the Medical Faculty, Gadjah Mada University, Yogyakarta.

\section{Measurements}

\section{EORTC QLQ-C30 Questionnaire}

We used the EORTC QLQ-C30 questionnaire, which is available in a validated Indonesian translation. ${ }^{10}$ This original questionnaire was developed by the EORTC for patients with all cancer types . The EORTC QLQ-C30 covers basic important personal dimensions in cancer patients, in the physical, psychological, and social domains. Furthermore, this questionnaire also covers cancer symptoms or cancer treatment-related symptoms such as nausea, vomiting, dyspnea, appetite loss, diarrhea, constipation, insomnia, as well as financial impact. The questionnaire has been used extensively in many countries to assess QoL of cancer in patients in their respective countries. $^{11}$ The normative data of Dutch population in female subjects were used as reference group $(\mathrm{n}=796){ }^{12}$ We used the Dutch population as the normative data in this study because the normative data of EORTC QLQ-C30 in Indonesian population are not available. The characteristics of Dutch subjects who filled in the EORTC QLQ-C30 questionnaire were female respondents $(\mathrm{n}=796)$ and a mean (SD) age of 50.8 (15) years. Around 63\% subjects reported the health problems as follows: heart disease, hypertension, asthma/chronic obstructive pulmonary disease, diabetes, depression, and joint disease.

\section{SF-36 Questionnaire}

The SF-36 is a generic instrument that can therefore be used in the general population and any group of patients 
with any illness as a generic QoL instrument. ${ }^{13}$ The SF-36 has QoL dimensions similar to the EORTC QLQ-C30, that is, physical, emotional/mental, social, pain, and vitality/energy. In previous studies that compared the SF-36 and EORTC QLQ-C30 in patients with cancer, they were found to be satisfactory psychometric instruments in assessing consequences of cancer on the physical, emotional, social, pain, and energy dimensions of the questionnaire. ${ }^{14}$ The normative data from the Dutch population aged 45 to 54 years old were used as reference data in this study $(\mathrm{n}=180) .{ }^{15}$ We used the Dutch population as the reference because of the collaboration study between the institutions in Indonesia and Netherlands and the normative data of SF-36 in Indonesia population are not available.

\section{Delayed Emesis}

Delayed emesis was defined as delayed nausea and vomiting as had to be reported by the patients in their diaries. Delayed nausea was measured by the Nausea Visual Analog Scale using a severity nausea scale of 0 to $100 .{ }^{16}$ Zero on the scale represents "no nausea" and a higher score represents "more severe delayed nausea." Patients were asked to indicate the number on the Nausea Visual Analog Scale once daily during a 4-day period after chemotherapy. The frequency of delayed vomiting was defined by asking patients to fill in a daily diary record of vomiting frequency. The vomiting episode was defined as single vomiting, and the next vomiting episode was defined as 1 minute after the previous vomiting episode. ${ }^{16}$ The delayed emesis was dichotomized into "response" and "no response" based on the patient's daily diary record. Patients were grouped into "response" if they did not experience nausea on 0 to 25 scores and did not vomit during 4 days after chemotherapy. Patients were grouped into "no response" if they experienced nausea and vomiting during the 4 days after chemotherapy. ${ }^{16}$

\section{Statistical Analysis}

Raw QoL scores were transformed into function scales, which range from 0 to 100. On the EORTC QLQ-C30, higher scales scores on the dimensions indicate better function, that is, better QoL. Higher scores in symptoms indicate more severe symptoms, that is, lower QoL. Symptoms scores are assessed with the EORTC QLQ-C30, whereas dimension scores of the functioning are measured with both EORTC QLQ-C30 and SF-36. Higher scores on the SF-36 indicate better QoL, except for fatigue and bodily pain where higher scores indicate more severe symptoms.

Descriptive data are presented as means and SDs. Differences of patients' QoL before and after chemotherapeutic treatment were analyzed using Student $t$ test. Differences in functions and scales between the 2 groups of delayed emesis were defined by an independent $t$ test. $P<0.05$ was considered statistically significant.

\section{RESULTS}

Patients' characteristics are summarized in Table 1. Of the 186 female cancer patients, $64.5 \%$ had cervical cancer, $27.4 \%$ had ovarian cancer, $4.3 \%$ had uterine cancer, and $3.8 \%$ patients were diagnosed with vulva cancer. The mean (SD)
TABLE 1. Patients' characteristics

\begin{tabular}{|c|c|}
\hline Age $(\mathrm{n}=181)$, mean $(\mathrm{SD}), \mathrm{y}$ & $48.3(19.8)$ \\
\hline \multicolumn{2}{|l|}{ Education $(\mathrm{n}=181), \mathrm{n}(\%)$} \\
\hline No education & $60(32.3)$ \\
\hline Elementary school & $50(26.9)$ \\
\hline High school & $64(34.4)$ \\
\hline Bachelor/diploma & $12(6.5)$ \\
\hline \multicolumn{2}{|c|}{ Type of gynecologic cancer $(n=186), n(\%)$} \\
\hline Cervical cancer & $120(64.5)$ \\
\hline Ovarian cancer & $51(27.4)$ \\
\hline Uterine cancer & $8(4.3)$ \\
\hline Vulva cancer & $7(3.8)$ \\
\hline \multicolumn{2}{|l|}{ Comorbidity $(\mathrm{n}=186), \mathrm{n}(\%)$} \\
\hline None & $156(83.9)$ \\
\hline 1 disease & $29(15.6)$ \\
\hline 2 diseases & $1(0.5)$ \\
\hline \multicolumn{2}{|c|}{ Stage of cervical cancer $(\mathrm{n}=120), \mathrm{n}(\%)$} \\
\hline Limited to pelvic & $88(73.3)$ \\
\hline Advanced stage & $32(26.7)$ \\
\hline \multicolumn{2}{|c|}{ Stage of ovarian cancer $\left(\mathrm{n}=50^{*}\right), \mathrm{n}(\%)$} \\
\hline Limited to pelvic & $31(62.0)$ \\
\hline Advanced stage & $19(38.0)$ \\
\hline \multicolumn{2}{|c|}{ Stage of uterine cancer $\left(\mathrm{n}=13^{*}\right), \mathrm{n}(\%)$} \\
\hline Limited to pelvic & $2(25.0)$ \\
\hline Advanced stage & $3(37.0)$ \\
\hline \multicolumn{2}{|l|}{ Stage of vulva cancer, n (\%) } \\
\hline Limited to pelvic & $1(14.3)$ \\
\hline Advanced stage & $6(85.7)$ \\
\hline
\end{tabular}

age of patients was 48.3 (19.8) years. Most of the patients had graduated from high school (34.4\%), whereas $32.3 \%$ of patients did not have formal education. Comorbidity was present in $15.6 \%$ of patients with 1 disease and $0.5 \%$ of patients with 2 diseases.

The available cytostatic drugs for the patients in this hospital were cisplatin, cyclophosphamide, doxorubicin, and 5-fluorouracil. In the cervical cancer group, most of the patients who had limited pelvic cervical cancer $(73.3 \%)$ were treated with cisplatin as a single agent or in combination with 5-fluorouracil, although this is not a standard schedule. The dose of cisplatin as a single agent was $70 \mathrm{mg} / \mathrm{m}^{2}$ every 3 weeks, whereas the dose of cisplatin was $50 \mathrm{mg} / \mathrm{m}^{2}$ in combination with $500 \mathrm{mg} / \mathrm{m}^{2}$ of 5-fluorouracil every week. In the ovarian cancer group with limited stage $(62.0 \%)$, the treatment consisted of $500 \mathrm{mg} / \mathrm{m}^{2}$ of cyclophosphamide, $50 \mathrm{mg} / \mathrm{m}^{2}$ of adriamycin, and $50 \mathrm{mg} / \mathrm{m}^{2}$ of cisplatin every 3 weeks.

The baseline QoL scores of all subjects are listed in Table 2. We compared the baseline QoL in these data with the reference studies. ${ }^{12,15}$ For this comparison, we have relied on 
TABLE 2. Comparison of baseline patients' QoL in this study and the reference studies

\begin{tabular}{|c|c|c|c|}
\hline & Baseline of This Study & Baseline of the Reference Data ${ }^{12,15}$ & $P$ \\
\hline EORTC QLQ-C30 & $\mathrm{n}=186$ & $\mathrm{n}=796$ & \\
\hline General QoL & $60(15)$ & $77(18)$ & $<\mathbf{0 . 0 0 1}$ \\
\hline Physical function & $77(20)$ & $89(17)$ & $<\mathbf{0 . 0 0 1}$ \\
\hline Role function & $68(25)$ & $87(22)$ & $<\mathbf{0 . 0 0 1}$ \\
\hline Emotional function & $94(12)$ & $88(17)$ & 0.004 \\
\hline Cognitive function & $95(10)$ & $92(16)$ & $<\mathbf{0 . 0 0 1}$ \\
\hline Social function & $72(23)$ & $93(18)$ & $<\mathbf{0 . 0 0 1}$ \\
\hline Pain & $33(25)$ & $18(24)$ & $<\mathbf{0 . 0 0 1}$ \\
\hline Fatigue & $25(21)$ & $20(21)$ & $<\mathbf{0 . 0 0 5}$ \\
\hline Nausea vomiting & $5(13)$ & $3.9(13)$ & 0.300 \\
\hline Dyspnea & $5(14)$ & $7.6(18)$ & $<\mathbf{0 . 0 0 1}$ \\
\hline Sleeping difficulty & $32(30)$ & $17(26)$ & $<\mathbf{0 . 0 0 1}$ \\
\hline Appetite loss & $18(25)$ & $4.4(14)$ & $<\mathbf{0 . 0 0 1}$ \\
\hline Constipation & $13(24)$ & $6.5(17)$ & $<\mathbf{0 . 0 0 1}$ \\
\hline Diarrhea & $4(26)$ & $3.8(14)$ & 0.942 \\
\hline Financial difficulty & $48(48)$ & $3.6(13)$ & $<\mathbf{0 . 0 0 1}$ \\
\hline SF-36 & $\mathrm{n}=186$ & $\mathrm{n}=180$ & \\
\hline General health perceptions & $52.9(15.2)$ & $71.6(23.0)$ & $<\mathbf{0 . 0 0 1}$ \\
\hline Physical function & $51.1(30.6)$ & $79.9(24.7)$ & $<\mathbf{0 . 0 0 1}$ \\
\hline Physical role functioning & $30.0(40.9)$ & $78.9(37.0)$ & $<\mathbf{0 . 0 0 1}$ \\
\hline Emotional role functioning & $32.8(43.5)$ & $83.6(33.1)$ & $<\mathbf{0 . 0 0 1}$ \\
\hline Mental health & $63.2(22.8)$ & $76.7(19.6)$ & $<\mathbf{0 . 0 0 1}$ \\
\hline Social function & $54.1(22.0)$ & $86.1(21.8)$ & $<0.001$ \\
\hline Bodily pain & $58.2(27.5)$ & $80.5(26.7)$ & $<\mathbf{0 . 0 0 1}$ \\
\hline Fatigue & $61.2(18.9)$ & $67.5(20.3)$ & 0.002 \\
\hline Health change & $42.8(28.7)$ & $51.9(19.8)$ & $<\mathbf{0 . 0 0 1}$ \\
\hline
\end{tabular}

Values are means (SD); bold $P$ values show the significant differences of baseline Qol scores between this study and the reference studies.

a publication ${ }^{12}$ for which the raw data were not available for us. As a result, it was not possible to adjust this comparison for confounding factors with a multivariate analysis. In the EORTC QLQ-C30, the functions score of this present study in both of the questionnaires were lower than those in reference studies, except for emotional function and cognitive function. Meanwhile, the symptoms scores such as pain, fatigue, nausea and vomiting, sleeping difficulty, appetite loss, and constipation were higher than those of reference studies. The significant differences of function scores were shown by all functions and symptoms, except for emotional function, nausea and vomiting, and diarrhea $(P<0.001)$. Moreover, the Indonesian cancer patients faced higher financial difficulty than the Dutch population. In the SF-36, the Indonesian cancer patients showed significantly lower functions and symptoms than those of the reference study $(P<0.05)$.

Seven patients died in this study during the delayed phase due to their cancer. Most patients (74.9\%) experienced delayed emesis in the 4 days after chemotherapy, that is, a response to the attempt to prevent nausea and vomiting. The patients' functions in the baseline and after chemotherapy based on the patients' response are presented in Figure 1. At baseline, no significant differences between the response group and the no-response group were found for any of the QoL domains and symptoms scale.

On the EORTC QLQ-C30, the patients' functions did not change much or even deteriorated after the chemotherapy. Fatigue was experienced by both response group and no-response group patients (Fig. 1). Meanwhile, in the SF-36, general health perceptions, emotional function, and social function were improved after chemotherapy. Figure 2 shows the patients' symptoms at baseline and after chemotherapy based on the response and the no-response groups. This figure shows that the nonresponse patients experienced more severe dyspnea, sleeping difficulty, appetite loss, and constipation after chemotherapy than at baseline.

The impact of delayed emesis on QoL dimensions in patients with and those without a response is presented in Table 3. Significant differences between the 2 groups in symptoms, such as fatigue, nausea, vomiting, and appetite loss, were found on the EORTC QLQ-C30 questionnaire. The no-response patients experienced more severe symptoms than response 


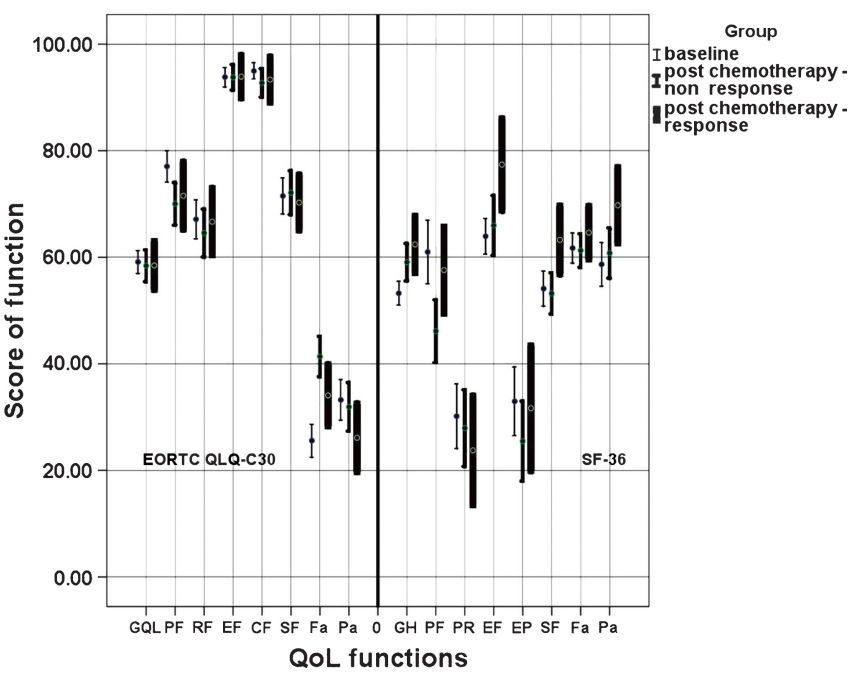

FIGURE 1. Scores on the EORTC QLQ-C30 and SF-36 at baseline and after chemotherapy in the response and no-response groups. CF indicates cognitive function; $E F$, emotional function; $E P$, emotional problem; $\mathrm{Fa}$, fatigue; $\mathrm{GH}$, general health; $\mathrm{GQL}$, general QoL; $\mathrm{Pa}$, pain; PF, physical function; PR, physical role; RF, role function; SF, social function.

patients in whom the attempted prevention of nausea and vomiting was effective, according to the diary data. Significant differences between the 2 groups on QoL dimensions, such as physical function, mental function, social function, and bodily pain, were also found using the SF-36 questionnaire. The dimensions score show that the no-response patients had lower QoL than the response patients. This study also found that both the response group and the no-response group showed the score deterioration in most of the dimensions and experienced worse symptoms compared with the baseline score

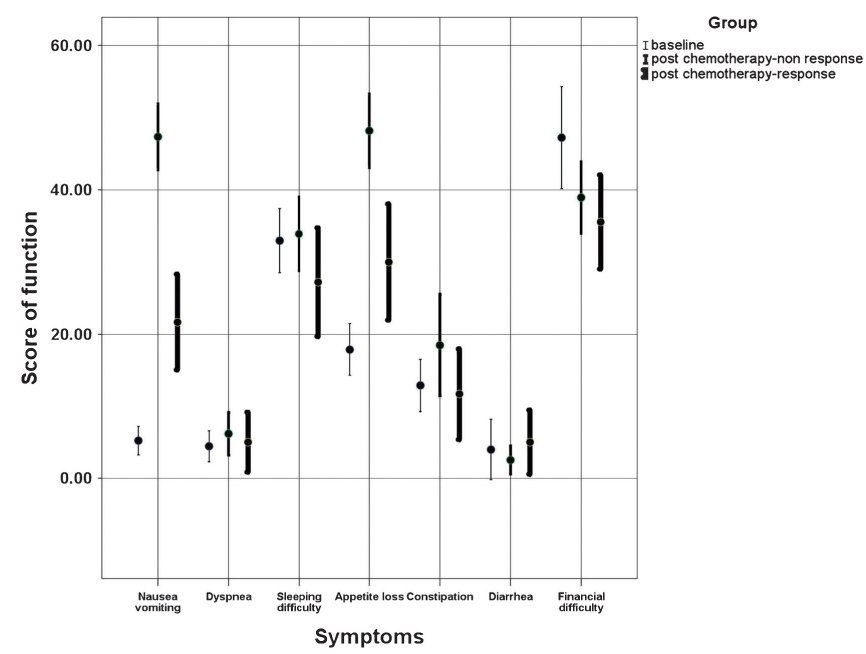

FIGURE 2. Symptoms on the EORTC QLQ-C30 at baseline and after chemotherapy for the response and no-response groups.
TABLE 3. Quality of life functions 5 days after chemotherapy based on delayed emesis

\begin{tabular}{|c|c|c|c|}
\hline & $\begin{array}{c}\text { Response } \\
\text { Group } \\
(n=45)\end{array}$ & $\begin{array}{c}\text { No-Response } \\
\text { Group } \\
(n=134)\end{array}$ & $P$ \\
\hline \multicolumn{4}{|l|}{ EORTC QLQ-C30 } \\
\hline General QoL & $58.4(17.5)$ & $58.4(16.4)$ & 0.979 \\
\hline Physical function & $71.6(24.3)$ & $70.0(22.1)$ & 0.673 \\
\hline Role function & $66.6(24.2)$ & $64.5(24.8)$ & 0.590 \\
\hline Emotional function & $93.9(15.3)$ & $93.7(13.2)$ & 0.956 \\
\hline Cognitive function & $93.3(16.3)$ & $92.7(14.7)$ & 0.799 \\
\hline Social function & $70.3(19.9)$ & $72.1(22.7)$ & 0.592 \\
\hline Pain & $26.1(24.6)$ & $31.9(25.1)$ & 0.142 \\
\hline Fatigue & $34.1(22.2)$ & $41.3(20.9)$ & 0.032 \\
\hline Nausea vomiting & $21.7(25.8)$ & $47.3(25.2)$ & $<0.001$ \\
\hline Dyspnea & $5.0(16.0)$ & $6.2(16.2)$ & 0.650 \\
\hline Sleeping difficulty & $27.2(29.1)$ & $33.9(28.2)$ & 0.140 \\
\hline Appetite loss & $30.0(31.1)$ & $48.2(28.0)$ & $<0.001$ \\
\hline Constipation & $11.7(24.4)$ & $18.5(38.9)$ & 0.218 \\
\hline Diarrhea & $5.0(17.2)$ & $5.0(34.6)$ & 0.993 \\
\hline Financial difficulty & $35.6(25.2)$ & $38.9(27.2)$ & 0.423 \\
\hline \multicolumn{4}{|l|}{ SF-36 } \\
\hline $\begin{array}{l}\text { General health } \\
\text { perceptions }\end{array}$ & $62.4(20.5)$ & $59.1(19.3)$ & 0.121 \\
\hline Physical function & $57.6(31.4)$ & $46.1(32.4)$ & 0.025 \\
\hline $\begin{array}{l}\text { Physical role } \\
\text { function }\end{array}$ & $23.8(39.4)$ & $27.9(39.6)$ & 0.504 \\
\hline $\begin{array}{l}\text { Emotional role } \\
\text { function }\end{array}$ & $31.7(45.3)$ & $25.5(41.3)$ & 0.362 \\
\hline Mental health & $77.4(33.1)$ & $65.9(30.9)$ & 0.024 \\
\hline Social function & $63.3(24.6)$ & $53.2(21.3)$ & 0.005 \\
\hline Bodily pain & $69.8(27.4)$ & $60.8(25.9)$ & 0.034 \\
\hline Fatigue & $64.6(19.1)$ & $61.3(17.4)$ & 0.241 \\
\hline Health change & $57.9(33.1)$ & $51.1(24.9)$ & 0.287 \\
\hline
\end{tabular}

Values are means (SD).

Significant score differences between the response and noresponse groups are indicated in bold.

(Table 3). Other factors that could have a negative impact on patients' QoL are the patients' characteristics such as comorbidity, cancer diagnosis, and stage of cancer. Meanwhile, only comorbidity showed significant impact on the physical function, pain, and fatigue symptoms (data are not presented).

\section{DISCUSSION}

Our results show that, despite optimal initial prophylactic use of antiemetics followed by suboptimal prophylaxis for delayed nausea and emesis, most patients with gynecologic cancer experience delayed emesis. We have to take into account that the standard of prophylactic antiemetic drugs 
that are used at our hospital is suboptimal. Despite this shortcoming, we decided to study the relationship of CINV and QoL.

A previous study showed that around $70 \%$ of patients receiving chemotherapy in a community hospital experienced delayed emesis. ${ }^{17}$ The present study shows a similar percentage of subjects experiencing delayed emesis (ie, 74.9\%). All patients were treated by a standard antiemetic regimen consisting of ondansetron and dexamethasone 1 hour before the cisplatin treatment. Because of the cost of further ondansetron with dexamethasone and certainly aprepitant, suboptimal therapy with oral metoclopramide was prescribed for delayed emesis/vomiting. On the basis of the international guidelines, ondansetron or granisetron in combination with dexamethasone on days 2 to 5 should be given after a highly emetogenic chemotherapy to prevent delayed emesis. ${ }^{7}$ All of our patients were treated with metoclopramide for 5 days, after cisplatin treatment. Only $34.3 \%$ patients had complete response in the delayed phase. Poor control of patients' symptoms after chemotherapy interfered our study patients' QoL. In another study, the efficacy of metoclopramide was increased significantly by the augmentation of dexamethasone. ${ }^{18}$

The delayed emesis in our patient sample led to a significant negative impact on the patients' QoL. Significant impact was illustrated by more severe symptoms, such as fatigue, nausea, vomiting, appetite loss, and pain after chemotherapy. Interestingly, the patients who did not experience delayed emesis also showed a deterioration of QoL. The scores of the various QoL dimensions in EORTC QLQ-C30 questionnaire were lower than those in reference groups before the chemotherapy treatment. The symptom scores were also higher than those in the reference groups. This probably indicates that Indonesian gynecologic cancer patients have lower QoL and experience more severe symptoms than the same groups in the Netherlands before start of chemotherapy, possibly related to the suboptimal treatment of delayed nausea/ emesis. A recent study in France suggested that the deterioration of physical function, role function, and general health could be a reason for impaired QoL in newly diagnosed cancer patients. ${ }^{19}$

The present study showed that delayed emesis affects patients' symptoms such as fatigue, appetite loss, and bodily pain. The higher score of the functions in the response group and the significant differences of function scores between the patients of the response group and the no-response group indicate that delayed emesis interferes with patients' daily function. Both response and no-response groups showed that CINV interferes with patients' QoL. These findings are similar to other studies of cancer patients treated with moderately to highly emetogenic chemotherapy, despite the use of antiemetic drugs. In addition, acute CINV affects patients' QoL even for patients who do not experience nausea or vomiting during the delayed phase. ${ }^{20}$ The patients' comorbidity should be considered as well as a factor that can affect physical function, pain, and fatigue.

In general, patients' QoL was decreased in our study after chemotherapy compared with baseline. These findings are similar to another study in a community oncology setting across the United States, which revealed that CINV significantly interferes with patients' QoL. ${ }^{1}$ Our study included cancer patients who received their first chemotherapy course with various emetogenic chemotherapy schedules.

General health perception, emotional, and social functions were maintained. This could be explained by the role of family and neighborhood support. One characteristic of the Indonesian society, especially in the rural area, is that many people come to the patient's house to give psychological support when the patient comes back from the hospital. This finding is supported by Noonan ${ }^{20}$ who reported that patients' QoL was not only affected by the symptoms of cancer and the adverse effect of treatment but also by the psychosocial condition, such as family support. The study of survivorship in cancer patients suggested that the ability to return to family, social, and work activities was an essential part of survivorship. $^{21}$

Another study that used the Functional Living Index of Emesis (FLIE) as the QoL instrument to study the relationship between CINV and patients' QoL revealed that the score of FLIE after chemotherapy decreased significantly, in the range of $21.6 \%$ to $24.4 \%$. Patients with CINV also had a decrease of health utility in the range of $15 \%$ to $6.9 \% .^{22}$ In addition, it has been reported that both acute and delayed emesis have a significant impact on patients' daily functioning. Furthermore, it is frequently underreported and untreated because the patients experience these symptoms after they have left the hospital. ${ }^{1}$ In our hospital, a similar situation exists because patients leave the hospitals, on average, 4 hours after the chemotherapy has been given.

Different health care providers in the Western world have variously predicted the incidence rates of CINV, and their prediction in prescription of antiemetics has also varied. For example, in patients receiving highly emetogenic chemotherapy, the use of 5-hydroxytryptamine receptor antagonists and dexamethasone as antiemetics led to an underestimation by the health providers in predicting delayed emesis. ${ }^{23}$ In contrast, the use of aprepitant in combination with dexamethasone and 5-hydroxytryptamine receptor antagonists led to health care providers' overestimation in predicting delayed CINV. Furthermore, it has been suggested that the health care providers increase their appreciation of delayed $\mathrm{CINV}$ incidence by using structured patient-reported outcome instruments. 5

On the basis of the results in the present study, we also recommend that the health care providers in Indonesia should closely monitor delayed emesis and prescribe an appropriate antiemetic prophylaxis.

The results of our study indicate that poor control of delayed emesis in cancer patients treated by highly emetogenic chemotherapy unnecessarily reduces the patients' QoL. Thus, appropriately potent antiemetics should be used to prevent delayed emesis. In clinical practice, the oncologist who prescribes chemotherapy in combination with suboptimal antiemetic prophylactic should be aware of delayed CINV because the delayed emesis adversely affects patients after they have left the hospital. Furthermore, the delayed emesis should be closely monitored to improve the patients' QoL and patients' adherence in following the next cycles of chemotherapy. Cognitive-behavioral interventions, counseling, and supportive therapy seem to be additional promising 
strategies to improve gynecologic cancer patients' QoL and their survivorship. ${ }^{1,21}$

\section{CONCLUSIONS}

Patients with gynecologic cancer in our study experienced severe symptoms, such as fatigue, nausea, vomiting, appetite loss, and pain after chemotherapy, despite adequate prophylactic use of antiemetics for acute nausea and vomiting but with insufficient prophylactic antiemetic therapy for chronic nausea and vomiting. These symptoms affected other domains as shown in both the EORTC QLQ-C30 and the SF-36 questionnaires.

Poor control of patients' chronic nausea and vomiting after chemotherapy has a negative impact on patients' QoL.

\section{ACKNOWLEDGMENT}

The authors thank Wendy Young who improved the English of the article.

\section{REFERENCES}

1. Cohen L, de Moor CA, Eisenberg P, et al. Chemotherapy-induced nausea and vomiting: incidence and impact on patient quality of life at community oncology settings. Support Care Cancer. 2007; 15:497-503.

2. Navari RM. Antiemetic control: toward a new standard of care for emetogenic chemotherapy. Expert Opin Pharmacother. 2009;10:629-644.

3. Trigg ME, Higa GM. Chemotherapy-induced nausea and vomiting: antiemetic trials that impacted clinical practice. J Oncol Pharm Pract. 2010;16:233-244.

4. Perwitasari DA, Gelderblom H, Atthobari J, et al. Anti-emetic drugs in oncology: pharmacology and individualization by pharmacogenetics. Int J Clin Pharm. 2011;33:33-43.

5. Majem M, Moreno ME, Calvo N, et al. Perception of healthcare providers versus patient reported incidence of chemotherapy-induced nausea and vomiting after the addition of NK-1 receptor antagonists [published online ahead of print November 18, 2010]. Support Care Cancer.

6. Melville A, Eastwood A, Kleijnen J, et al. Management of gynaecological cancers. Qual Health Care. 1999;8:270-279.

7. Kris MG, Hesketh PJ, Somerfield MR, et al. American Society of Clinical Oncology guideline for antiemetics in oncology: update 2006. J Clin Oncol. 2006;24:2932-2947.

8. Penson RT, Wenzel LB, Vergote I, et al. Quality of life considerations in gynecologic cancer. FIGO 26th Annual Report on the Results of Treatment in Gynecological Cancer. Int J Gynaecol Obstet. 2006;95:S247-S257.

9. Available at: http://globocan.iarc.fr/. Accessed October 3, 2010.
10. Perwitasari DA, Atthobari J, Dwiprahasto I, et al. Translation and validation of EORTC QLQ-C30 into Indonesian version for cancer patients in Indonesia. Jpn J Clin Oncol. 2011; 41:519-529.

11. Koller M, Aaronson NK, Blazeby J, et al. Translation procedures for standardised quality of life questionnaires: the European Organisation for Research and Treatment of Cancer (EORTC) approach. Eur J Cancer. 2007;43:1810-1820.

12. van de Poll-Franse LV, Mols F, Gundy CM, et al. Normative data for the EORTC QLQ-C30 and EORTC-sexuality items in the general Dutch population. Eur J Cancer. 2011;47: 667-675.

13. Ware JE Jr, Gandek B. Overview of the SF-36 Health Survey and the International Quality of Life Assessment (IQOLA) Project. J Clin Epidemiol. 1998;51:903-912.

14. Apolone G, Filiberti A, Cifani S, et al. Evaluation of the EORTC QLQ-C30 questionnaire: a comparison with SF-36 Health Survey in a cohort of Italian long-survival cancer patients. Ann Oncol. 1998;9:549-557.

15. van der Zee KI, Sanderman R. Rand-36. Manual. Groningen, The Netherlands: Noordelijk Centrum voor Gezondheidsvraagstukken; 1993.

16. Double-blind, dose-finding study of four intravenous doses of dexamethasone in the prevention of cisplatin-induced acute emesis. Italian Group for Antiemetic Research. J Clin Oncol. 1998;16:2937-2942.

17. Hilarius DL, Kloeg PH, van der Wall E, et al. Chemotherapy-induced nausea and vomiting in daily clinical practice: a community hospital-based study [published online ahead of print January 22, 2011]. Support Care Cancer. doi: 10.1007/s00520-010-1073-9.

18. Bhatia A, Tripathi KD, Sharma M. Efficacy \& tolerability of ondansetron compared to metoclopramide in dose dependent cisplatin-induced delayed emesis. Indian J Med Res. 2004; 120:183-193.

19. Boini S, Briancon S, Guillemin F, et al. Impact of cancer occurrence on health-related quality of life: a longitudinal pre-post assessment. Health Qual Life Outcomes. 2004;2:4.

20. Noonan K. The impact of chemotherapy induced nausea vomiting on the daily function and quality of life of patients. Adv Stud Nurs. 2005;3:16-21.

21. Biegler KA, Chaoul MA, Cohen L. Cancer, cognitive impairment, and meditation. Acta Oncol. 2009;48:18-26.

22. Lachaine J, Yelle L, Kaizer L, et al. Chemotherapy-induced emesis: quality of life and economic impact in the context of current practice in Canada. Support Cancer Ther. 2005;2:181-187.

23. Grunberg SM, Deuson RR, Mavros P, et al. Incidence of chemotherapy-induced nausea and emesis after modern antiemetics. Cancer. 2004;100:2261-2268. 